\title{
Rapid Tooling Method for Soft Customized Removable Oral Appliances
}

\author{
Mika Salmi $^{1{ }^{1},}$, Jukka Tuomi ${ }^{1}$, Rauno Sirkkanen ${ }^{1}$, Tuula Ingman $^{2}$ and Antti Mäkitie ${ }^{1,3}$ \\ ${ }^{I}$ Department of Industrial Engineering and Management, BIT Research Centre, Aalto University, School of Science, \\ Espoo, Finland \\ ${ }^{2}$ Department of Oral and Maxillofacial Diseases, Helsinki University Central Hospital and University of Helsinki, \\ Helsinki, Finland \\ ${ }^{3}$ Department of Otorhinolaryngology - Head and Neck Surgery, Helsinki University Central Hospital and University of \\ Helsinki, Helsinki, Finland
}

\begin{abstract}
Traditionally oral appliances i.e. removable orthodontic appliances, bite splints and snoring / sleep apnea appliances are made with alginate impressions and wax registrations. Our aim was to describe the process of manufacturing customized oral appliances with a new technique i.e. rapid tooling method. The appliance should ideally be custom made to match the teeth. An orthodontic patient, scheduled for conventional orthodontic treatment, served as a study subject. After a precise clinical and radiographic examination, the approach was to digitize the patient's dental arches and then to correct them virtually by computer. Additive manufacturing was then used to fabricate a mould for a soft customized appliance. The mould was manufactured using stereolithography from Somos ProtoGen O-XT 18420 material. Casting material for the mould to obtain the final appliance was silicone. As a result we managed to create a customized soft orthodontic appliance. Also, the accuracy of the method was found to be adequate. Two versions of the described device were manufactured: one with small and one with moderate orthodontic force. The study person also gave information on the subjective patient adaptation aspects of the oral appliance.
\end{abstract}

Keywords: Additive manufacturing, computer-aided design (CAD), computer-aided manufacturing (CAM), rapid prototyping, oral appliance, orthodontics.

\section{INTRODUCTION}

Traditionally the protocol to fabricate oral appliances i.e. removable orthodontic appliances, bite splints and snoring / sleep apnea appliances, includes alginate impressions and wax registrations taken by the dentist and the appliance made by the dental technician. Three-dimensional computeraided design (3D-CAD) creates new possibilities in this field allowing greater use of industrially manufactured appliances while respecting the biological tissue reaction in the dental tissues [1]. Computer-aided graphical 3D-reconstruction has been used to trace the prenatal development of the human temporomandibular joint [2]. The first and still best known CAD / Computer Aided Manufacturing (CAM) technology in odontology was Cerec (Siemens, Germany) to manufacture ceramic inlays [3]. Clear orthodontic aligners provide a way to move the teeth with an aesthetic removable appliance in patients with only minor orthodontic problems or the aligner can also be used as a finishing or retention appliance.

Lin [4] introduced a method where a clear and hard tooth aligner is first manufactured by digitizing teeth, and then straightening them virtually by computer, and further

*Address correspondence to this author at the Aalto University, School of Science, Department of Industrial Engineering and Management, BIT Research Centre P.O. Box 15500 FI-00076 Aalto, Finland; Mobile +358 50512 2746; Fax +358 9470 23665;

E-mail: mika.salmi@aalto.fi additive manufacturing of a corrected pattern for a vacuumheat process. Lee et al. [5] used a combination of computed tomography and rapid prototyping to manufacture a physical copy of unusual tooth root anatomy. Lauren et al. [6] used a computer-assisted method for design and fabrication of hard occlusal splints. They scanned teeth from stone casts and virtually adjusted them. Splints were manufactured by milling. Joffe [7] used 60 commercially available hard clear appliances, which were made by the following method: digitalizing tooth, virtual straightening, additive manufacturing a mould, pressure forming and finishing. Keski-Nisula et al. [8] investigated a soft eruption guidance appliance for children and found it as an effective method to restore normal occlusion and eliminate the need for further orthodontic treatment.

Kohorst et al. [9] measured the accuracy of different CAM systems and the best mean value was $58 \mu \mathrm{m}$ and the worst ones 183 and $206 \mu \mathrm{m}$. Germani et al. [10] investigated the accuracy of a scanning plaster model and additive manufactured plaster replica. They tested four different digitizing systems and eight different combinations of additive manufacturing technology with various materials. Metzger et al. [11] combined computerized tomography imaging and optical plaster model scanning to obtain a virtual model with accurate teeth and jaws for occlusal control. They also undertook virtual repositioning of the jaws and then printed physical models of the splint with a 3D printer. 
Table 1. Requirements for Method

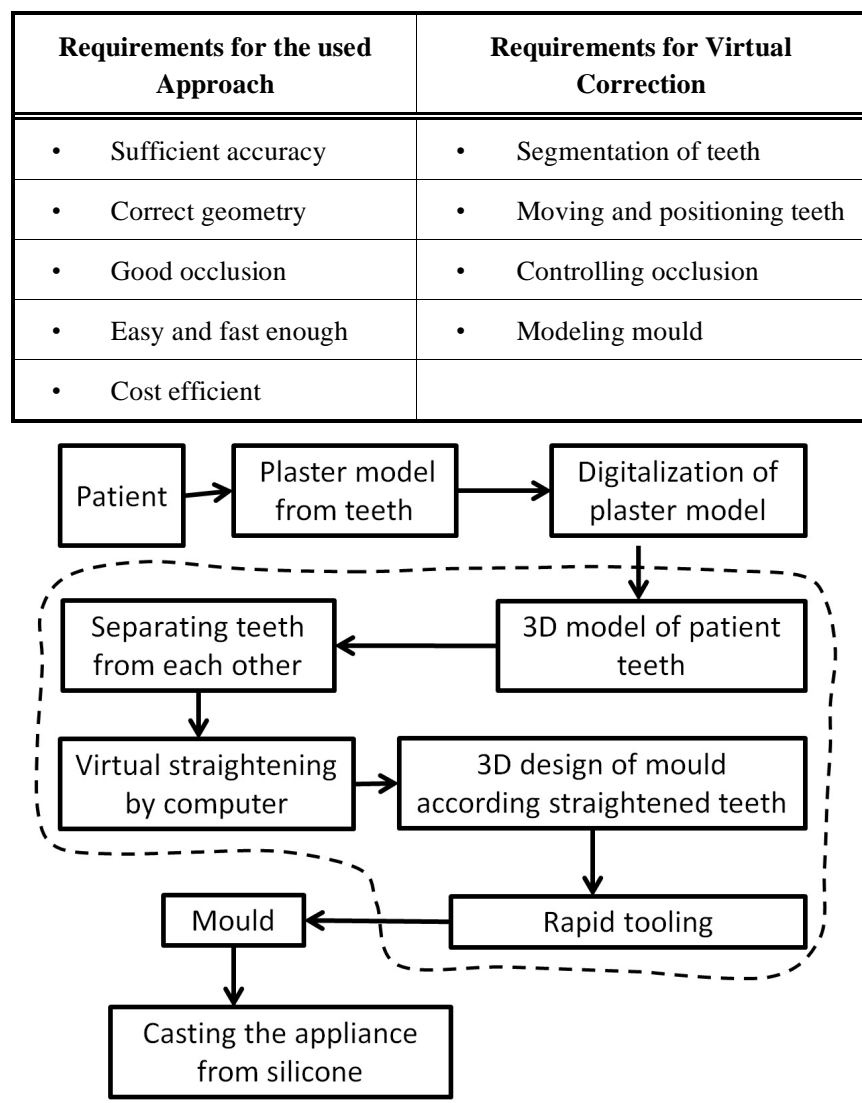

Fig. (1). Process for planning and manufacturing of customized orthodontic appliances. The dashed line defines the digital steps of the process.

The aim of the present study was to generate a custommade oral appliance with a new technique i.e. a rapid tooling method. It is a process using additive manufacturing to directly fabricate a mould quickly and economically. A patient, planning to start conventional orthodontic treatment, was asked to give information regarding their adaptation, convenience and tolerance to the oral appliance made by this technique. After a precise clinical and radiographic examination, the approach was used to digitize the patient's dental arches and then to correct them virtually by computer to develop a custom made removable oral appliance. Silicone was used as the casting material for the mould. Two versions of the device were manufactured: one with small and another with moderate correcting force.

\section{MATERIALS AND METHODS}

Several methods to create an unfixed, customized and soft oral appliance were investigated and used. Our approach was to use $3 \mathrm{D}$ digitizing to create a $3 \mathrm{D}$ model for virtual orthodontic correction. The established requirements for the used study design are presented in Table $\mathbf{1}$.

Using ideal virtual teeth positioning a mould for silicon casting was designed and additive manufacturing was used to create it. The mould was then used to cast the appliance from silicone. A flow chart of the process is demonstrated in Fig. (1).

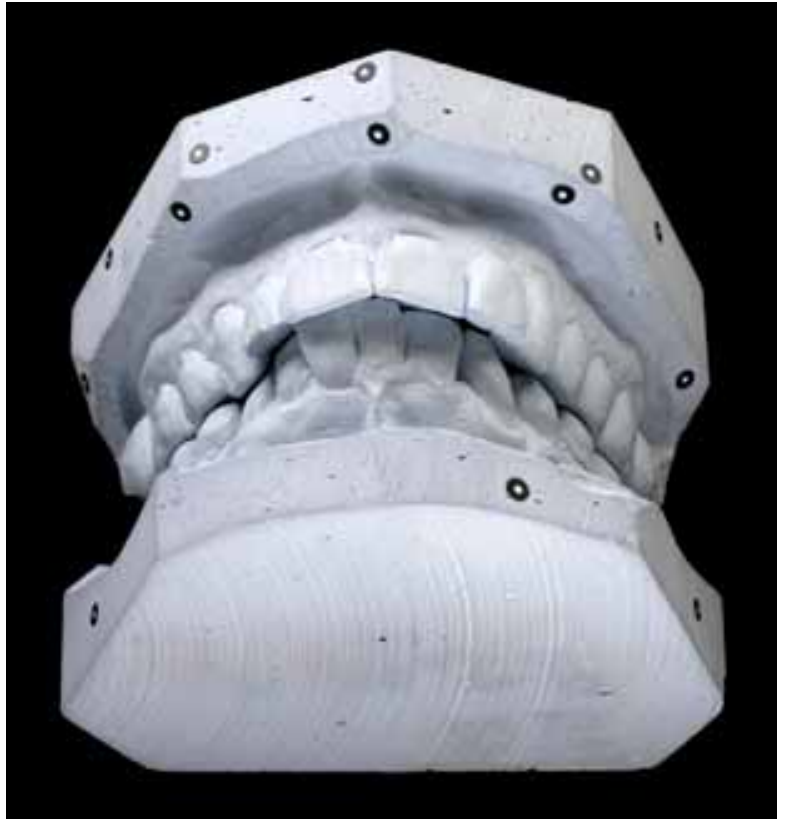

Fig. (2). The plaster model.

\section{EXPERIMENTAL PATIENT FOR APPLIANCE TESTING}

The study patient, who was asked to test the convenience of the appliance use, was a 46-year-old healthy female, with no medication or allergies. The patient had no previous orthodontic treatment. The dental and periodontal tissues were healthy, as assessed by careful clinical and radiological examination. Due to temporary pain in the temporomandibular joints and bruxism, the patient used a bite splint nightly. In the orthodontic examination, the convexity of the profile was normal, mesiodistal molar relationship was ideal (Angle class I) on the right and cusp-cusp on the left side, horizontal overjet was $4 \mathrm{~mm}$ and vertical overbite $1 / 3$ of the crown height. Upper and lower right first premolars had been extracted earlier. There was minor crowding of the upper canine area. The canines were palatally inclined, on both right and left sides as well as on the lower incisor area, which were labially inclined (Fig. 2). According to the clinical and radiological examination, the patient was suitable for the experimental use of the appliances. Each appliance was tested for two minutes.

\section{Digitization of Teeth}

The teeth digitizing can be either direct or indirect. The direct method comprises imaging or scanning of the dental arches straight from the patient's mouth and generating the 3D model from these data. In the indirect method a plaster model is first made from the teeth and then digitized by using a scanner or other imaging techniques.

At imaging the slice thickness should be as small as possible and the patient should not move during the imaging. Additional artefacts can cause errors. These error sources can ruin the $3 \mathrm{D}$ model and therefore, a direct method with imaging is not suitable for all patients. Imaging allows for including the roots of the teeth into the 3D model and segmenting the teeth one by one, assuming that sufficient accuracy exists. 


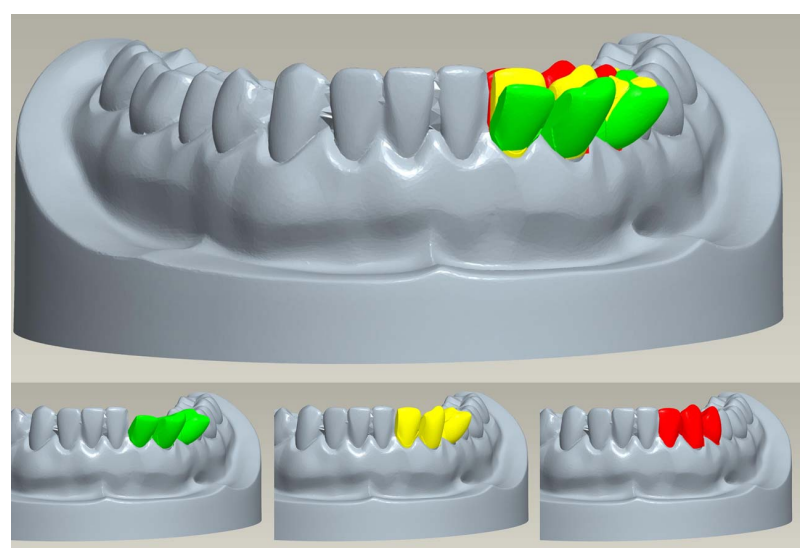

Fig. (3). Digital moving and repositioning of teeth in green, the moderate correction in yellow and the high force correction in red.

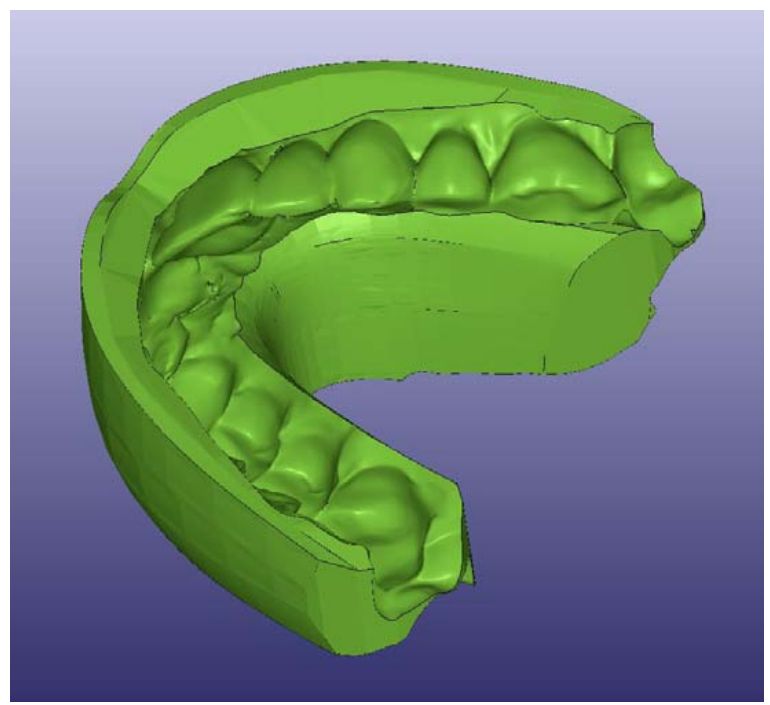

Fig. (4). 3D-model of orthodontic appliance.

The teeth were digitized by first taking a plaster model from the patient's teeth and used the GOM ATOS 3D scanner (GOM GmbH, Germany) to obtain a virtual 3D model from the plaster model. It is important to use software that is able to fix small holes and errors in the obtained scanned image, because these can cause interference with the Boolean operations in the 3D design of mould. We separated teeth from each other by using the basic cutting tools in the Viscam RP 4.0 software (Marcam Engineering GmbH, Germany).

\section{Virtual Alignment}

The digital data of the separated teeth were imported into Pro Engineer Wildfire 4.0 software (Parametric Technology Corp, USA). Each corrected tooth was a separate data set and these were combined in an assembly mode. The software allowed the teeth to be movedto get an optimal dental arch form. Fig. (3) demonstrates removing and repositioning of the teeth. The red colour represents an oblique position, the yellow the intermediate, and the green the final ideal position.

\section{D Design of the Mould}

Viscam RP 4.0 software was used for the mould design. A previous 3D model of an orthodontic appliance with no

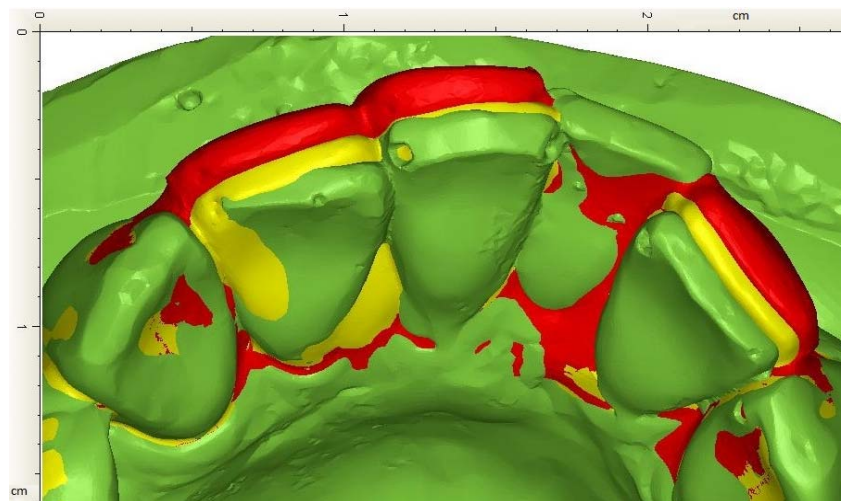

Fig. (5). Current lower jaw with teeth in green, the moderate correction in yellow and the high force correction in red.

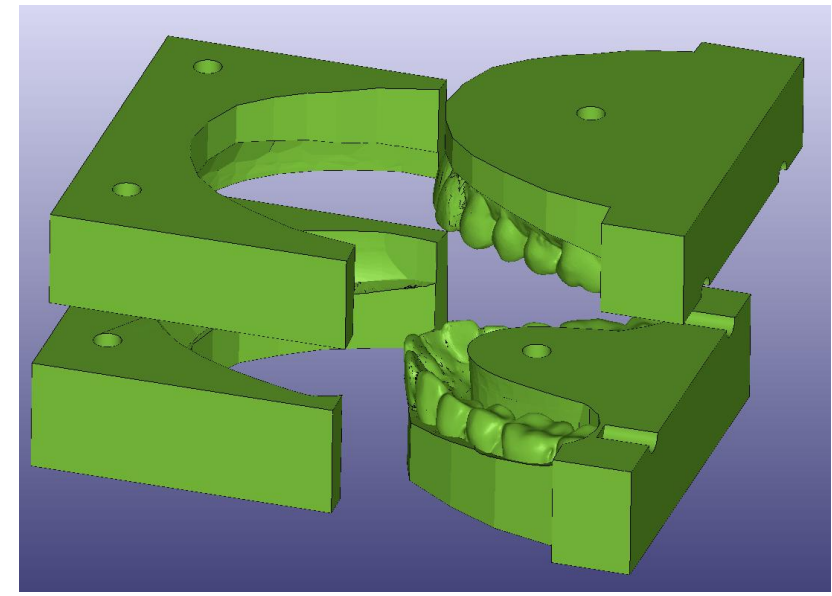

Fig. (6). Four-piece mould for the appliance.

geometry data of the teeth was used as a starting point. This formed the geometry for the outer shape of the appliance. The inner geometry to the appliance was achieved by using Boolean cutting operation with aligned upper and lower teeth and this produced the final geometry for the appliance. The 3D model of the orthodontic appliance is shown in Fig. (4). As a slab for the mould a rectangular prism was used. The mould's actual final form was obtained by a Boolean cutting operation and using the shape of the appliance.

Guide pins were placed to align parts of the mould to each other and a channel was constructed for squeezing silicon into the mould, but these were not used during the actual manufacturing phase. The modelling could also have been done by making a parametric model which allows an adjustment of the mould geometry according to the imported corrected 3D model.

In the second version the teeth were adjusted more to get more force applied to them from the appliance. Over correction was $1-3 \mathrm{~mm}$ and this seemed to produce too much force. The current lower dental arch with corrections is presented in Fig. (5).

Also, the two-piece mould was changed to a four-piece mould because of difficulties in removing the appliance from the mould due to undercuts. With the four-piece mould (Fig. 6) removing the appliance from the mould was relatively easy. 


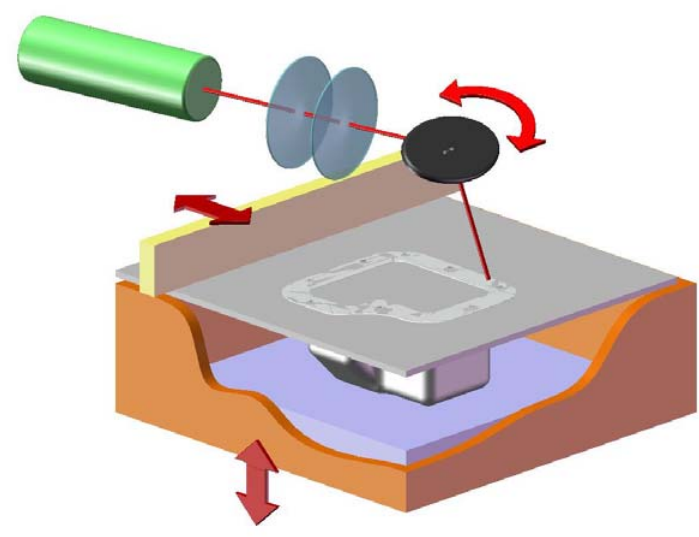

Fig. (7). A schematic drawing of the stereolithography.

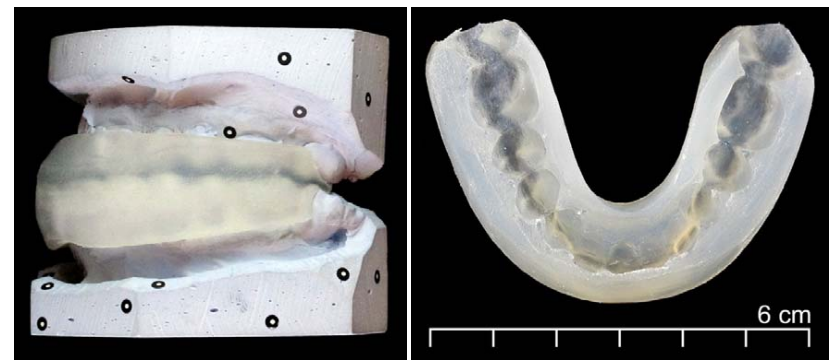

Fig. (8). Appliance between plaster models and the finished orthodontic appliance.

\section{Manufacturing}

The mould was manufactured by using stereolithography, which is an additive manufacturing technology, where parts are built layer by layer by curing photopolymer with ultraviolet laser. The laser beam traces a part cross-section pattern on the surface of the liquid resin on each layer and between layers the building platform descends by a single layer thickness. A schematic figure of the stereolithography process is shown in Fig. (7). Used machine was SLA 350 (3D Systems, USA). For the material Somos ProtoGen O-XT 18420 (DSM Functional Materials, USA) was chosen because it has a very low material shrinkage and it can stand the temperatures needed in the casting phase. The layer thickness used was $0.05 \mathrm{~mm}$. After manufacturing the mould was placed in a post-cure apparatus for 60 minutes. Silicone was used as casting material.

\section{Inspection}

After manufacturing we used GOM ATOS II 3D scanner (GOM mbH, Germany) to capture virtual 3D model from manufactured appliance. Before measuring the silicone was sprayed with mixture of titanium oxide and alcohol to gain better response for optical scanner. Comparison was performed using GOM Inspect V7 SR2 (GOM mbH, Germany).

\section{RESULTS}

Fig. (8) depicts the appliance between plaster models and a finished appliance. Both manufactured customized soft oral appliances were tested in the patient's mouth repeatedly for two minutes each to get some subjective feedback of various aspects regarding its use (ease, comfort and convenience).

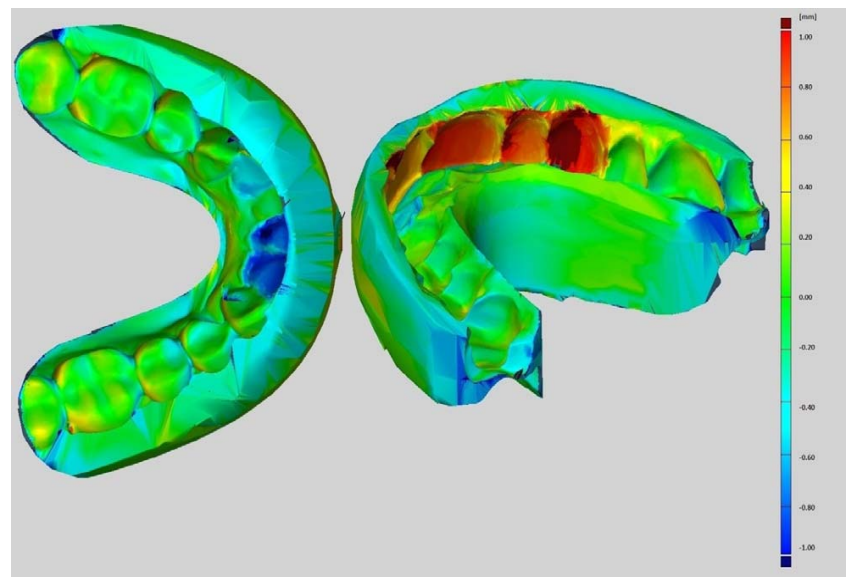

Fig. (9). Accuracy of appliance compared with the designed 3D model. Scale: red $+1.0 \mathrm{~mm}$ to blue $-1.0 \mathrm{~mm}$.

The device with moderate force was subjectively more efficient but also caused a slightly unpleasant sensation. The small force version did not create that much force sensation but was more comfortable to use. Furthermore, an exact fit and a user-friendly surface quality were other attributes for both versions reported by the patient. The true movement of teeth was not investigated since this effect was not the focus of the present study.

The accuracy of the system is visualized in Fig. (9). Maximum dimensional errors of approximately $1 \mathrm{~mm}$ were found at thin walls and sharp corners when comparing the physical model to the 3D design.

\section{DISCUSSION}

Custom-made brackets for orthodontic purposes are an ideal target for additive manufacturing techniques. In the present study scanned data of the patient's teeth were used to virtually design a mould for silicon casting. Furthermore, additive manufacturing was used to create the physical mould. The final appliance was made of silicone by a casting method using this mould.

While performing the digitization phase, the jaws must be in the same position as they would be when using the appliance. This is to prevent possible unwanted pain in the temporomandibular joint or the bite muscles or changes in the occlusion. It also makes the 3D modeling easier because the modeler does not have to reset the jaws or to consider any forces due to biting. An alternative option is to combine three scans: upper and lower jaw as well as occlusion. These appliances have a limited ability to align teeth due to their inability to create tooth rotations, intrusion or root movement. The appliance might also be useful in finishing orthodontic treatment after fixed appliances. At present only a sparse literature regarding this issue exists. Thus, the complex force delivery properties of the appliance system have to be systematically investigated and studied until commercial use can be considered.

The advantage of these removable oral appliances made by the rapid tooling method compared to conventional appliance production protocol might be a faster production technique, as well as more inexpensive and more precise oral appliances. The geometric dimensions of the custom-made 
appliance were found to be accurate compared to the 3D model. In soft appliances, though, the accuracy of the geometric dimensions is not that crucial since the material is pliable compared to hard appliances. The need for cheaper oral appliances is greater among snorers or in patients with sleep apnea.

One method for manufacturing is to use a soft slab that is for example thermally formed over rapid manufactured straightened teeth. However, tipping movements are predictable with thermoplastic appliances and it is much more difficult to establish a comparable amount of root control [12]. Also the thickness of the appliance as well as the material used for the appliance may have a significant influence on the amount and type of the orthodontic tooth movement [1315].

The aim of the present study was to describe the multidisciplinary process that is needed to additive manufacture a custom-made soft orthodontic appliance. Evidence of clinical applicability of this created appliance is needed before further commercial production. However, production of a physical prototype usually leads to further developing phases of the product before any clinical experience can be obtained.

Selection of the patients to the orthodontic treatment demands accurate diagnosis, and the number of suitable patients might be fewer due to a multitude of orthodontic problems in many patients, i.e. skeletal problems, hypodontia, etc. In the future, during the use of the oral appliance manufactured by rapid tooling method for either orthodontic, sleep apnea, temporomandibular joint or bruxism problems, a careful follow-up of the effects of the appliance as well as the changes in the occlusion should be investigated in a controlled manner.

\section{CONCLUSIONS}

The customized soft orthodontic appliance made from silicone could be manufactured by making a mould with stereolithography from Somos ProtoGen O-XT 18420 material. The appliance with moderate force was more efficient but caused a slightly unpleasant sensation. The small force version did not create that much force but was more comfortable to use. Both appliances were well tolerated and convenient to use.

\section{ACKNOWLEDGEMENT}

This work was supported by Tekes, the Finnish Funding Agency for Technology and Innovation, DeskArtes Oy, EOS Finland Oy, Inion Oy, Planmeca Oy, LM-Instruments Oy and Helsinki University Hospital Research Funds. The authors would like to thank M.Sc. (Tech.) Pekka Paavola for the photographs. This study was part of the BIOMAN II research project.

\section{CONFLICT OF INTEREST}

None declared.

\section{REFERENCES}

[1] Vassura G, Vassura M, Bazzacchi A, Gracco A. A shift of force vector from arm to brain:3D computer technology in orthodontic treatment management. Int Orthod 2010; 8: 46-63.

[2] Radlanski RJ, Lieck S, Bontschev NE. Development of the human temporomandibular join. Computer-aided 3D-reconstructions. Eur J Oral Sci 1999; 107: 25-34.

[3] Pallasen U, Van Dijken JWV. An 8-year evalution of sintered ceramic and glass ceramic inlays processed by the Cerec CAD/CAM system. Eur J Oral Sci 2000; 108: 239-46.

[4] Lin AC. Integration of $3 \mathrm{D}$ CAD, reverse engineering and rapid prototyping in fabrication of invisible tooth aligner. Syst Man Cybern, IEEE Int Conf 2005; 3: 2431-6.

[5] Lee SJ, Jang KH, Spangberg LS, et al. Three-dimensional visualization of a mandibular first molar with three distal roots using computer-aided rapid prototyping. Oral Surg Oral Med Oral Pathol Oral Radiol Endod 2006; 101: 668-74.

[6] Lauren M, McIntyre F. A new computer-assisted method for design and fabrication of occlusal splints. Am J Orthod Dentofacial Orthop 2008; 133: S130-5.

[7] Joffe L. Current Products and Practice Invisalign®: early experiences. J Orthod 2003; 30: 348-52.

[8] Keski-Nisula K, Hernesniemi R, Heiskanen M, Keski-Nisula L, Varrela J. Orthodontic intervention in the early mixed dentition: A prospective, controlled study on the effects of the eruption guidance appliance. Am J Orthod Dentofacial Orthop 2008; 133: 25460 .

[9] Kohorst P, Brinkmann H, Li J, Borchers L, Stiesch M. Marginal accuracy of four-unit zirconia fixed dental prostheses fabricated using different computer aided desing/computed-aided manufacturing systems. Eur J Oral Sci 2009; 117: 319-25.

[10] Germani M, Raffaeli R, Mazzolini A. A method for performance evalution of RE/RP systems in dentistry. Rapid Prototyping J 2010; 16: 345-55.

[11] Metzger MC, Hohlweg-Majert B, Schwarz U, Teschner M, Hammer B, Schmelzeisen R. Manufacturing splints for orthognathic surgery using a three-dimensional printer. Oral Surg Oral Med Oral Pathol Oral Radiol Endod 2008; 105: 1-7.

[12] Hahn W, Zapf A, Dathe H, et al. Torquing an upper central incisor with aligners-acting forces and biomechanical principles. Eur J Orthod 2010; 32: 607-13.

[13] Barbagallo LJ, Shen G, Jones AS, Swain MV, Petocz P, Darendeliler MA. A novel pressure film approach for determining the force imparted by clear removable thermoplastic appliances. Ann Biomed Eng 2008; 36: 335-41.

[14] Hahn W, Fialka-Fricke J, Dathe H, et al. Initial forces generated by three types of thermoplastic appliances on an upper central incisor during tipping. Eur J Orthod 2009; 31: 625-31.

[15] Hahn W, Dathe H, Fialka-Fricke J, et al. Influence of thermoplastic appliance thickness on the magnitude of force delivered to a maxillary central incisor during tipping. Am J Orthod Dentofacial Orthop 2009; 136: 1-7.

Received: February 22, 2012

Revised: April 12, 2012

Accepted: April 12, 2012

(C) Salmi et al.; Licensee Bentham Open.

This is an open access article licensed under the terms of the Creative Commons Attribution Non-Commercial License (http://creativecommons.org/licenses/by-nc/3.0/) which permits unrestricted, non-commercial use, distribution and reproduction in any medium, provided the work is properly cited. 